\title{
NUMBER OF HOURS OF SLEEP WITH CHANGES IN BLOOD PRESSURE AMONGST MEDICAL STUDENTS IN WESTERN MAHARASHTRA
}

\author{
Dimple Manoj Tarwani ${ }^{1}$, Kunkulol RR ${ }^{2}$, Sohan Chaudhari ${ }^{1}$, Chinmaye Batwal ${ }^{3}$, Liz Thaliath ${ }^{3}$ \\ ${ }^{I}$ MBBS student, ${ }^{2}$ Professor Department of Pharmacology, ${ }^{3}$ Research Associate, Rural Medical College, \\ Pravara Institute of Medical Sciences, Loni, Ahmednagar, Maharashtra, India.
}

\begin{abstract}
Introduction: Sleep has become one of the most ignored factors today. More sleep or less sleep does not matter it is just compensated the next day. Importance of regular and timely sleep amongst the students and their correlation with blood pressure is necessary to be found out so that the students can be made aware of and can be provided treatment to prevent further complications. Method: Demographic profile was noted it includes name, age, gender, professional year of MBBS and residence. History of sleeping was noted and other factors which influences sleeping pattern (i.e, alcohol, smoking, an association of dreams with sleep, midnight awakenings with sleep, caffeine intake before bed and average stress levels) through a well-structured questionnaire. Blood pressures of all eligible students were recorded 3 times in a week at the same time by the principal investigator with the same instrument according to the JNC 7 classification for hypertension. Results: Amongst all the medical students, $6 \%$ had systolic hypertension and $22.50 \%$ had diastolic hypertension. Comparing blood pressures according to sleeping patterns, amongst cases (less than 5 hours of sleep) $20.8 \%$ had systolic hypertension and $56.30 \%$ had diastolic hypertension. Whereas in controls (more than 5 hours of sleep) $1.40 \%$ had systolic hypertension and $11.90 \%$ had diastolic hypertension. Factors such as alcohol consumption, smoking, caffeine consumption before sleep, dreams, midnight awakenings and stress were found to be associated with sleeping patterns and the results were found to be extremely significant $(\mathrm{p}<0.0001)$ statistically. Conclusion: Sleep duration of less than 5 hours was noted in $24 \%$ of the total population. The subjects with lesser number of sleeping hours had increased levels of blood pressure and a higher prevalence of hypertension. The average systolic blood pressure in subjects with less than 5 hours of sleep (cases) showed $48.50 \%$ pre-hypertensives and $20.80 \%$ hypertensives whereas in case of average diastolic blood pressure $18.50 \%$ were prehypertensive and $56.30 \%$ were hypertensive.
\end{abstract}

Keywords: Blood pressures; Sleeping hours; Medical Students.

\section{INTRODUCTION}

Sleep is a condition of body and mind which typically recurs for several hours every night, in which the nervous system is inactive, the eyes are closed, the posture muscles relaxed, and consciousness practically suspended [1]. Blood pressure is the pressure of blood in the circulatory system, often measured for diagnosis since it is closely related to the force and rate of the heart beat and the diameter and elasticity of the arterial walls [2].

Typically, daily sleep duration has been declining among adults for more than a generation now, with median sleep duration falling from 8 hours/night in 1950's to 7 hours/night in recent years with more than one third now sleeping fewer than 7 hours/night [3].

It is well known that the students today have a very hectic and stressful lifestyle and medical students are no exception to the stress. Much of this reduction in sleep duration reflects voluntary sleep restriction, with nearly half of individuals reporting that they restrict sleep to watch TV, use the internet, work or studies. Short term

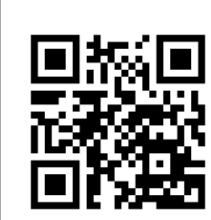

DOI: $10.31878 /$ ijcbr.2019.52.15

eISSN: 2395-0471

pISSN: 2521-0394 experimental sleep restriction for as little as one night has been reported to increase blood pressure in both healthy and hypertensive patients $[3,4]$.

Stress is a complex condition with emotional, cognitive and biological factors. Stress produces many neuroendocrine responses in the body, the most important being hypothalamus-pituitary-adrenal axis. The HPA axis is also related to the sleeping cycle. Thus, stress leads to decreased sleep which is known as stress related insomnia or physiological insomnia which is usually short term but produces significant health effects on one's mind and body [5].

On consideration of medical students, requirement of longer hours of practice and study time makes them compromise on their sleeping time. A huge bulk of syllabus and simultaneous practical which cann't be reduced, and other curricular and co-curricular activities leads to voluntary restriction of sleep in medical students. The effect of short sleep durations on hypertension may increase the risk of cardiovascular disease and mortality [6].

Daily consumption of 2 or more alcohol beverages tend to increase the sleep duration. This may lead to change in sleeping patterns [7,8]. The New York (Reuters Health) states that about 15 percent of surgeons have alcohol abuse and this rate is somewhat higher than the rest of the population. The effect of alcohol on sleep patterns is an additional factor to its morbidity potential.

Correspondence: Dimple Manoj Tarwani, MBBS student, Rural Medical College, Pravara Institute of Medical Sciences, Loni, Ahmednagar, Maharashtra. Email: director.research@pmt.pims.org 
Cigarette smoking has been associated with a high prevalence of sleep-related complains. Cigarette contains nicotine, which is a potent stimulant and decreases the sleep duration and hampers the quality of sleep. Caffeine works similarly as nicotine, it is a stimulator and disturbs the sleeping cycle.

Reports state that $70 \%$ of Indian students own a smartphone and $90 \%$ out of these are addicted to it. Gadget usage has been on a constant rise in our country especially amongst the youth. Using gadgets before sleeping reduces sleeping hours and increases midnight awakenings and hampers the quality of sleep [9].

In view of the afore mentioned effects of depressed sleep it was thought prudent to screen medical students of Rural Medical College situated in rural western Maharashtra for their sleeping habits and to find out its association with changes in blood pressure and comparing them based on various criteria. Hence a study was planned to prevent severe complications in later life and restore the health of future doctors.

Aims and Objectives: To find out the proportion of sleeping disorders amongst medical students, correlate number of sleeping hours with changes in blood pressure. To estimate the systolic and diastolic blood pressure of medical students and classify them according to the number of hours of sleep.

\section{MATERIAL AND METHODOLOGY}

Study design: Descriptive study

Ethics approval: Study was commenced after the approval from the ethical committee.

Place of research: Rural Medical College at Loni.

All the enrolled students are subjected to the following inclusion and exclusion criteria:

Inclusion criteria: All the students from all the professional years of MBBS studying

Exclusion criteria: Students not present on either day of the study, having a history of hypertension (family), congestive heart diseases, renal failure congenital anomalies and major Psychiatric disorders

Sample size: Random 100 students selected from each group (total two hundred)

\section{Methodology:}

Visits scheduled for data collection: Visit 1: Introduction to the project, obtaining consent and questionnaire. Visit 2-4: Measurement of blood pressure

Demographic profile: This includes name, age, gender, professional year of MBBS and residence

History: History of sleeping was noted and other factor which influences sleeping pattern (i.e, alcohol, smoking, association of dreams with sleep, midnight awakenings with sleep, caffeine intake before bed and average stress levels) through a well-structured questionnaire.

Sleeping pattern: All the medical students are first evaluated for average routine hours of sleep by giving a questionnaire where sleeping hours of 7 days is record- ed and an average was taken. For comparison of sleep duration and BP, students were divided to average routine sleep less than 5 hours of sleep (cases), whereas those with average routine sleep of more than 5 hours were considered (control).

Blood pressure: Blood pressures of all eligible students was recorded 3 times in a week at the same time by the principle investigator with the same instrument according to the Joint National Committee 7 (JNC 7) classification for hypertension [10].

\section{RESULTS}

In the present study total participants were 200 included (equal distribution of male and female), based on the academic year, there were $30 \%$ students from $I^{\text {st }}$ year, $7 \%$ from II $^{\text {nd }}$ year, $40 \%$ from III ${ }^{\text {rd }}$ year, $8 \%$ from final year and $15 \%$ were interns. According the sleeping time 152 students $(76 \%)$ were sleeping $>5$ hours and 48 students $(24 \%)$ were sleeping $<5$ hours.

Out of the total population $28 \%$ were alcoholics, $8.5 \%$ were smokers, $75.5 \%$ had dreams in the night, $37.5 \%$ of the total population experienced midnight awakenings (Midnight awakenings is factor which contributes to poor sleep quality).

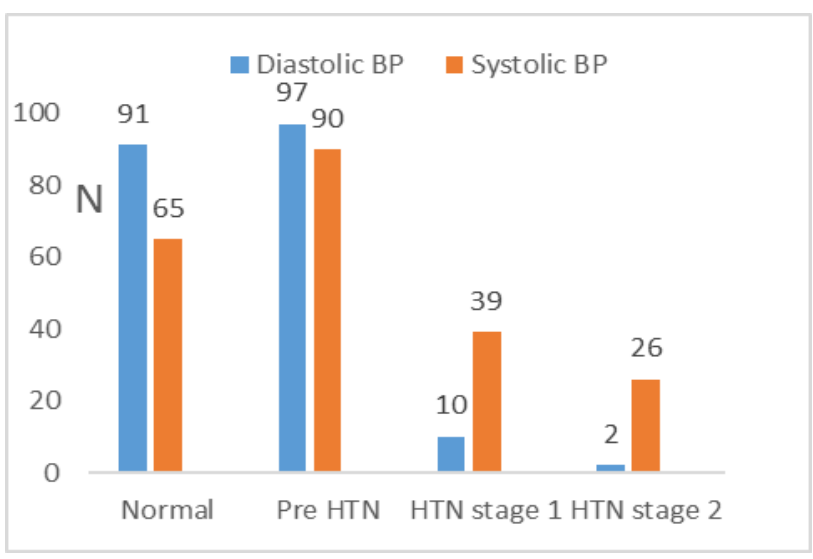

Fig 1: Classification of Diastolic \& systolic blood pressure in medical students

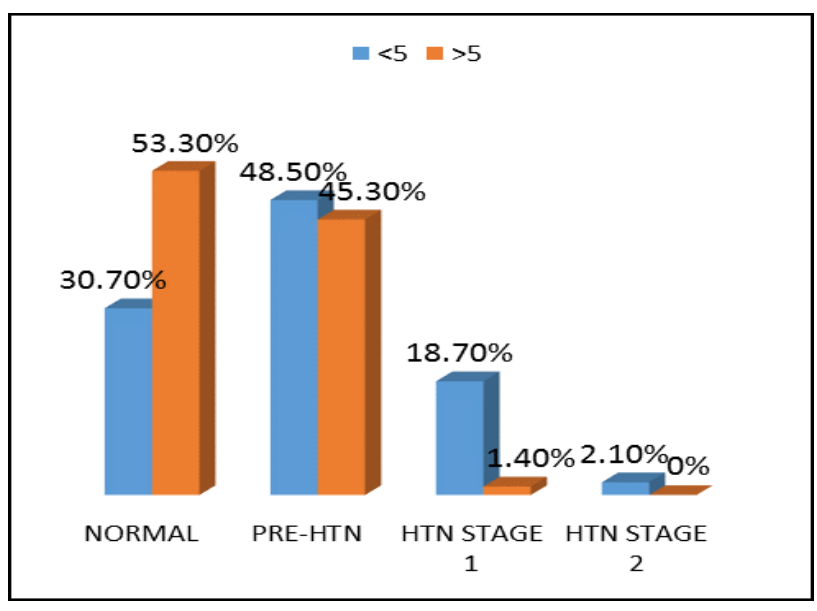

Fig 2: Classification of systolic blood pressure based on sleeping patterns 
Comparing the average systolic blood pressure there are maximum students in prehypertension stage i.e. $48.50 \%$ and $6 \%$ are hypertensives. There is a rise in number of hypertensives in diastolic blood pressure as compared to systolic blood pressure i.e. there are a total of $22.50 \%$ students with diastolic hypertension.

On comparison of average systolic blood pressure prehypertension, hypertension stage 1 and stage 2 are found to be more in cases i.e. in students sleeping less than 5 hours.

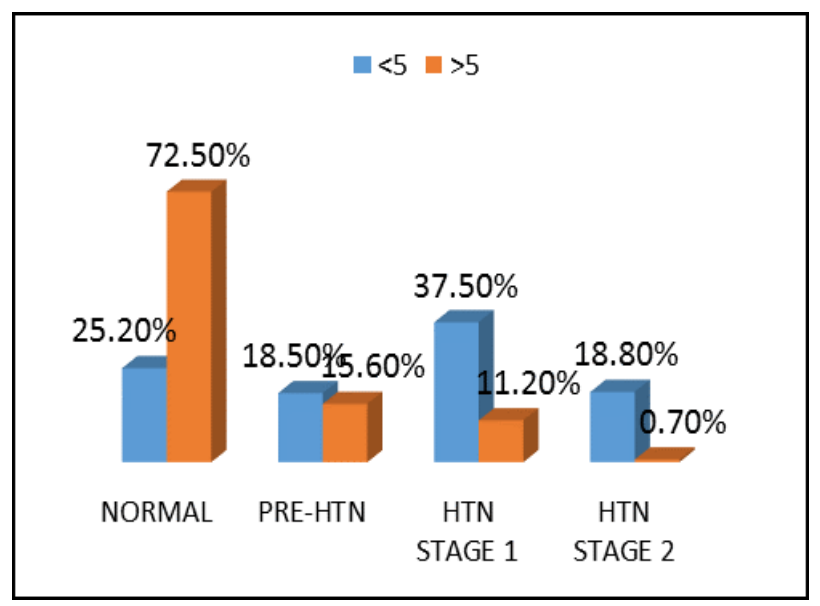

Fig 3: Classification diastolic blood pressure based on sleeping pattern

Based on average diastolic blood pressure, a total of $56.30 \%$ of cases were hypertensive whereas only $11.90 \%$ controls showed a similar trend.

\section{Factors influencing the seeping pattern}

The Fig 4 shows that alcoholics compared to nonalcoholics have lesser sleep duration. This comparison is statistically extremely significant $(\mathrm{p}<0.0001$; odd's ratio $=28.289$ )

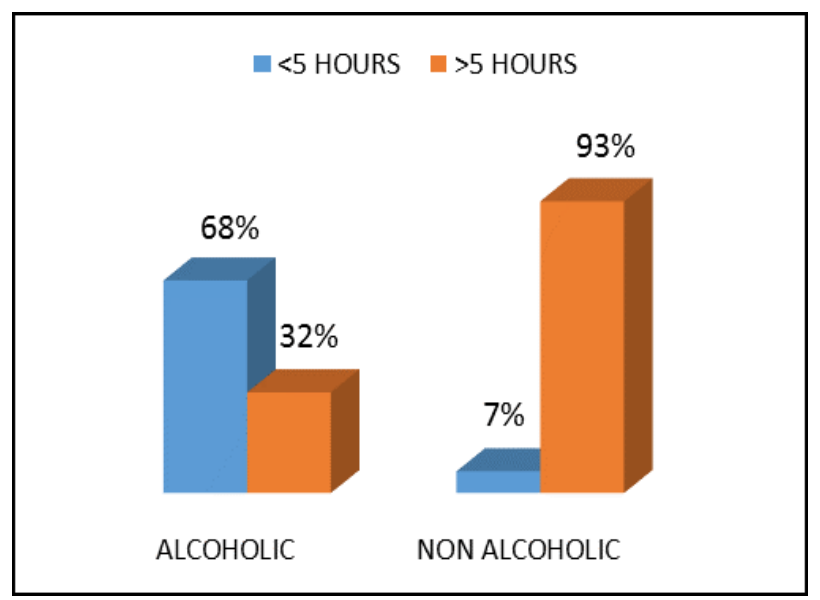

Fig 4: Association of alcohol with sleep

On comparing smokers and non-smokers, smokers have 13.74 times more chance of being sleep deprived (odd's ratio $=13.74)$.

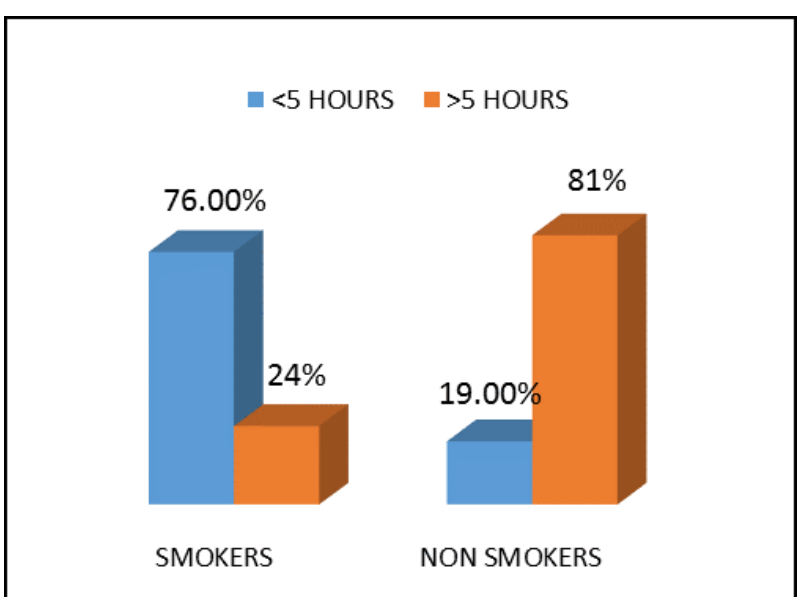

Fig 5: Association of smoking with sleep

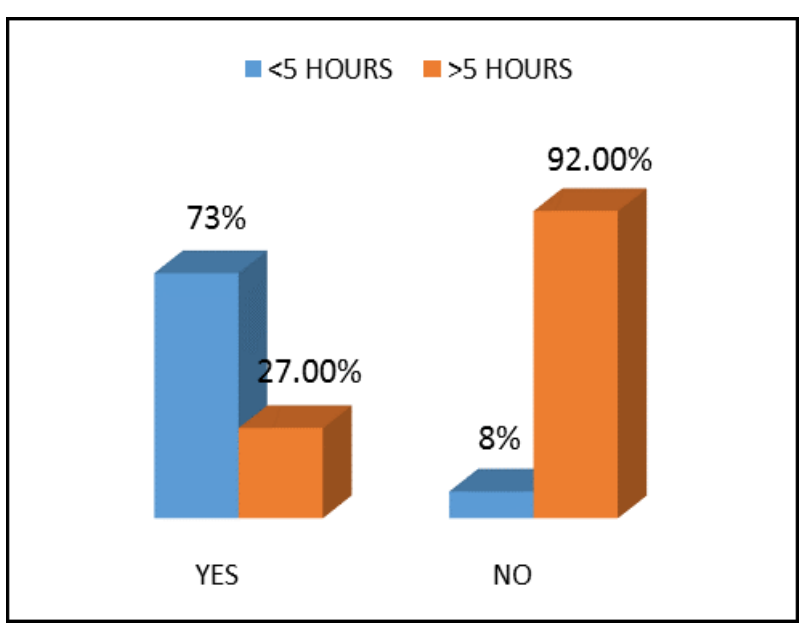

Fig 6: Association of dreams with sleep

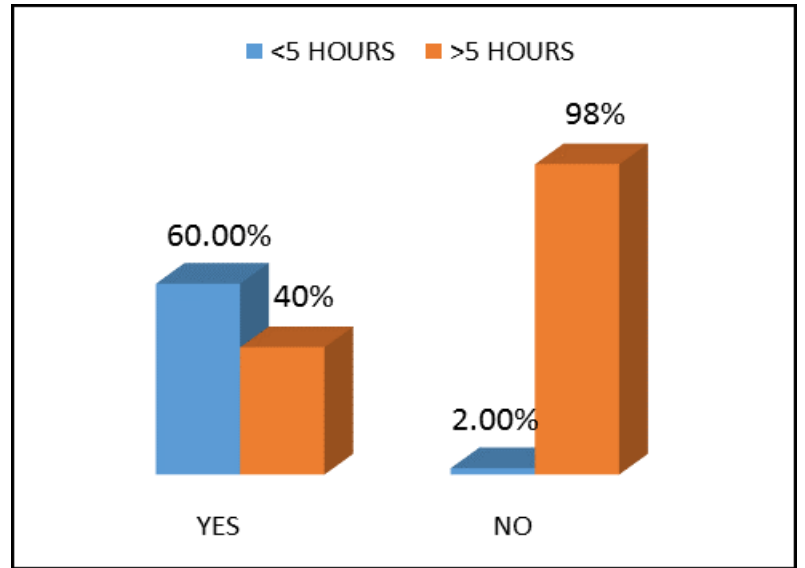

Fig 7: Association of midnight awakening

$73 \%$ of the subjects who dream while sleeping have shown less number of sleeping hours as compared to only $8 \%$ of subjects who do not dream have less sleep.

Amongst all the subjects having midnight awakenings only $40 \%$ slept more than 5 hours i.e. they could complete their sleep. Whereas those with no midnight awakenings $98 \%$ of them slept more than 5 hours. This comparison is statistically significant.

$71 \%$ of the subjects consuming caffeine before sleep slept for less number of hours as compared to only $20 \%$ 


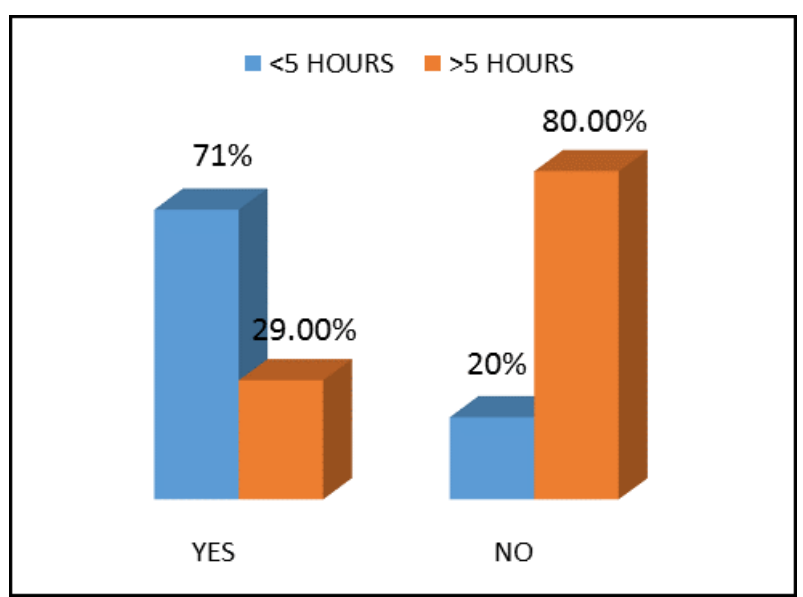

Fig 8: Association of caffeine consumption before sleep with sleep

of those who did not consume caffeine. About $8.50 \%$ population consumed caffeine just before going to bed mainly in the form of tea or coffee.

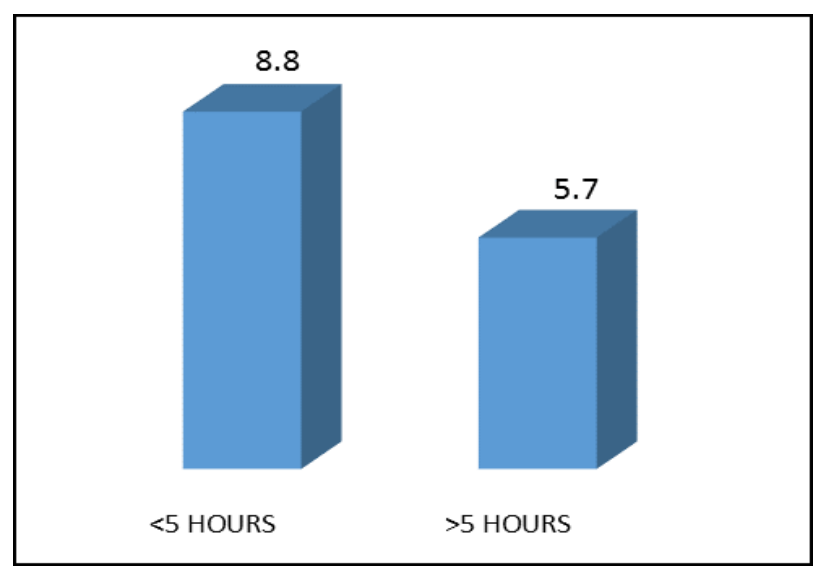

Fig 9: Association of average stress level with sleep

\section{DISCUSSION}

On classifying the students based on their blood pressures, $6 \%$ of the subjects had higher systolic blood pressure i.e. were hypertensive whereas $48.50 \%$ were prehypertensive. On the contrary $22.5 \%$ of the subjects had diastolic hypertension and $45 \%$ were prehypertensive on considering diastolic blood pressure.

On considering the number of sleeping hours, the subjects were divided into two groups i.e. more than 5 hours of sleep who were considered controls and less than 5 hours of sleep were considered cases. $24 \%$ of the subjects slept less than 5 hours whereas $76 \%$ of the subjects slept more than 5 hours. On comparing the blood pressures, both systolic and diastolic blood pressures were found to be on the higher side in cases which is in accordance to a study conducted by David A. Calhoun and Susan M Harding, who showed similar results of association of less sleep with increased blood pressures [1]. They also stated that activation of the hypothalamicpituitary-adrenal axis and the sympathetic nervous system as seen in insomnia may predispose to hypertension development. Sleep also alters the autonomic nervous function which leads to variations in blood pressures and mainly increases it.

As sleep affects the blood pressure, certain factors were also considered which may affect sleeping patterns especially in medical students. Out of the total population $28 \%$ consumed alcohol out which only $32 \%$ slept for more than 5 hours a day proving alcohol to be responsible for reduction in the sleeping quality and quantity. These results were in accordance to a study conducted by Subhajit Chakraborty et.al. who showcased similar results in his study on alcohol and sleeping patterns [11].

Amongst the smokers $76 \%$ had less than 5 hours of sleep as compared to only $19 \%$ amongst non-smokers. This huge hike in the subjects with a smaller number of sleeping hours can be attributed to nicotine which is a potent stimulant. Applying the statistical tests, the results were found to be very significant $(p<0.0001)$ and smokers had 13.743 times more chance disturbed sleeping patterns. Caffeine consumption just before sleep has also shown to lessen the number of sleeping hours. Only $8.50 \%$ of subjects consumed caffeine before sleeping out of which $71 \%$ had a smaller number of sleeping hours whereas out of the $91.50 \%$ subjects who did not consume caffeine before sleeping had only $20 \%$ population with reduced sleeping time. (Odd's ratio $=9.800)$

Occurrence of dreams is also considered as a factor which affects the number of sleeping hours. In my study, there were $24.50 \%$ subjects who used to get dreams whereas $75.50 \%$ subjects did not get dreams as in Table $12.73 \%$ of dreamers had short sleeping time whereas amongst the non-dreamers only $8 \%$ had shorter duration of sleep concluding dreams to shorten the sleep timespan. Midnight awakenings also majorly affect the sleeping time. According to my findings out of the $37.50 \%$ population with midnight awakenings $60 \%$ slept for less than 5 hours whereas only $40 \%$ slept for more than 5 hours. On the contrary $62.50 \%$ population who did not experience midnight awakenings only $2 \%$ slept for less than 5 hours, rest of the $98 \%$ had adequate sleep of more than 5 hours, signifying that midnight awakenings shortens the sleep span drastically. On statistical analysis people with midnight awakenings had 61 times more chance of shorter sleep duration.

Considering stress, the average stress levels were found to be higher in cases i.e. 8.8 as compared to controls which was 5.6. Therefore, it can be concluded that stress reduces the number of sleeping hours. These findings are in accordance to a study conducted Keum San Han et al. [5].

Dagmara Dimitriou, et al [2] in their study have stated that stimulant consumption and gadget usage before going to bed decreases the number of sleeping hours and hampers the quality of sleep. They also stated that decreased sleep can attributed as one of the causes of hypertension. Their findings are in accordance to my study which also proves the relation between a smaller number of sleeping hours and hypertension.

Subhajit et.al. in their study "Daytime sleepiness: Associations with alcohol use and sleep duration in Americans" [11] have found that alcoholics had a lower sleep duration in the night and the day as compared to people who consumed alcohol occasionally or did not consume 
it at all. They have also said that there is a possibility that after the alcohol is metabolised in the latter half of the night, the sleep is shallow and fragmented. This shallow and fragmented sleep can lead the subject to a state of sub-acute sleep deprivation with continued heavy drinking over time.

Lin Zhang et.al. in their study entitled "Cigarette smoking and nocturnal sleep architecture" [12] have stated that compared with never smokers, current smokers experience greater difficulty in initiating and maintaining sleep and are generally more dissatisfied with their sleep quality. They also attributed the causes to be: 1) Nicotine in cigarettes can stimulate the release of several neurotransmitters that collectively participate in regulating the sleep-wake cycle, 2) Habitual smokers often experience acute withdrawal as the intake of nicotine is curtailed during sleep, 3) The medical consequences associated with cigarette smoking, such as chronic obstructive lung disease, can disrupt sleep continuity and have a negative impact on sleep architecture.

$\mathrm{g}$ to complaints of day time sleepiness.

Keum San Han, Lin Kim and Insop Shim in their work entitled "Stress and sleep disorder" [5] have stated that excessive stress is detrimental on many levels to humans, and it activates the defence system of the central nervous system. Stress related physiological responses cause the endocrine responses and behavioural responses. They have also proved that stress related thinking relates to insomnia and poor quality of sleep.

In my study subjects with a smaller number of sleeping hours have shown a greater prevalence of hypertension. The smaller number of sleeping hours especially in medical students can be attributed to various causes mentioned earlier but one main factor which affects sleep the most is stress. All the other things are secondary like alcohol, smoking etc. which a person indulges into when he/she is stressed, be it academic, personal or professional stress. The present trend of keeping up late in the night for studies or social media has decreased the sleeping quality drastically making the students more prone to developing hypertension and thereby various cardiovascular and renal disorders.

\section{CONCLUSION}

Sleep duration of less than 5 hours was noted in $24 \%$ of the total population. The subjects with a smaller number of sleeping hours had increased levels of blood pressure and higher prevalence of hypertension. The average systolic blood pressure in subjects with less than 5 hours of sleep (cases) showed $48.50 \%$ pre-hypertensives and $20.80 \%$ hypertensives whereas in case of average diastolic blood pressure $18.50 \%$ were prehypertensive and $56.30 \%$ were hypertensive.

Suggestions: Though being in the medical profession very few students know about the correlation of sleep and blood pressure variations. This study recommends awareness of all the students from outside the medical colleges as well to know the importance of enough and good quality sleep. There is a need to know the importance of proper sleep as the youngsters today compromise on their sleep very frequently. Telling them the necessity of good sleep is the need of the hour as we need healthy and responsible doctors soon.
Funding: Research was supported by ICMR.

\section{REFERENCES}

[1] David A Calhoun, Susan M Harding. Sleep and Hypertension. Chest 2010;138(2):434-43

[2] Dagmara Dimitriou, Frances Le Cornu Knight, Patrick Milton. The role of Environmental Factors on Sleep Patterns and School Performance in Adolescents. Front Psychol, 2015;6:1717

[3] Kristen L Knutson, Eve Van Cauter, Paul J Rathouz, Lijing L Yan, Stephen B. Hulley, Kiang Liu, Diane S Lauderdale; Association between sleep and blood pressure in midlife: The Cardia Sleep Study. Arch Intern Med, 2009;8:169

[4] Wasim A Shaikh, Minal Patel, SK Singh. Association of sleep duration with arterial blood pressure profile of Gujarati Indian adolescents; Indian Journal of Public Medicine. 2010;35(1): 125-9

[5] Keum San Han, Lin Kim and Insop Shim. Stress and sleep disorder; Experimental Neurobiology. 2012;21(4):141-50

[6] Ines Paciencia, Henrique Barros, Joana Araujo, Elisabete Ramos; Association between sleep duration and blood pressure in adolescents; Hypertension Research. 2013;36:747-52.

[7] Rey-Lopez JP, De Carvalho HB. Sleep time and cardiovascular risk factors in adolescent: The Helena study. Sleep Medicine. 2014;15(1):104-10

[8] Buysse DJ, Reynolds III, Mon CF, Bermany TH, Kupfer SR. The Pittsburgh Sleep Quality Index : A New Instrument for Psychiatric Practice and Research. Journal of Psychiatric Research. 1989;28 (2), 193-213

[9] Harneet KW, Hong Li, Michael Rueschman, Deepak L Bhatt, Sanjay R Patel, Start F Quan, et al. Association of severe obstructive sleep apnea and elevated nlood pressure despite antihypertensive medication use. Journal of Clinical Sleep Medicine. 2014;10(08):193-213

[10] Bethesda, The Seventh Report of the Joint National Committee on Prevention, Detection, Evaluation, and Treatment of High Blood Pressure. National High Blood Pressure Education Program. National Heart, Lung, and Blood Institute (US); 2004 Aug. Available online https://www.ncbi.nlm.nih.gov/ books/NBK9633/

[11] Subhajit Chakraborty. Daytime sleepiness: Associations with alcohol use and sleep durations in Americans. Sleep disorders. 2014;Article ID 959152: 7

[12] Lin Zhang. Cigarette smoking and nocturnal sleep architecture; American Journal of Epidemiology. 2006;164(6):529-37. 